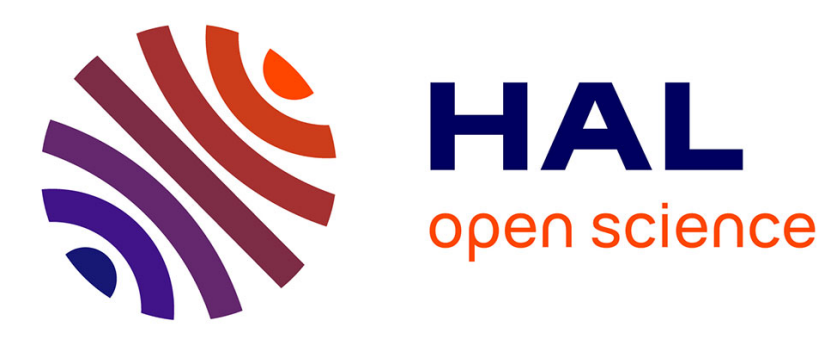

\title{
Actions affines isométriques propres des groupes hyperboliques sur des espaces $\ell \mathbf{p}$
}

Aurélien Alvarez, Vincent Lafforgue

\section{To cite this version:}

Aurélien Alvarez, Vincent Lafforgue. Actions affines isométriques propres des groupes hyperboliques sur des espaces $\ell$ p. Annales Scientifiques de l'École Normale Supérieure, 2018, 51 (nov.-déc.), pp.13891398. 10.24033/asens.2377 . hal-02406390

\section{HAL Id: hal-02406390 \\ https://hal.science/hal-02406390}

Submitted on 12 Dec 2019

HAL is a multi-disciplinary open access archive for the deposit and dissemination of scientific research documents, whether they are published or not. The documents may come from teaching and research institutions in France or abroad, or from public or private research centers.
L'archive ouverte pluridisciplinaire HAL, est destinée au dépôt et à la diffusion de documents scientifiques de niveau recherche, publiés ou non, émanant des établissements d'enseignement et de recherche français ou étrangers, des laboratoires publics ou privés. 


\title{
ACTIONS AFFINES ISOMÉTRIQUES PROPRES DES GROUPES HYPERBOLIQUES SUR DES QUOTIENTS D'ESPACES $\ell^{p}$
}

\author{
AURÉLIEN ALVAREZ ET VINCENT LAFFORGUE
}

\begin{abstract}
Résumé. Nous démontrons que tout groupe hyperbolique admet une action affine isométrique propre sur un quotient d'un espace de Banach $\ell^{p}$, pour tout $p>1$ suffisamment proche de 1.
\end{abstract}

\section{Point de déPart}

Dans un travail précédent [AL16], nous donnions une nouvelle démonstration élémentaire et auto-contenue d'un théorème de $\mathrm{Yu}$ : tout groupe hyperbolique admet une action affine isométrique propre sur un espace $\ell^{p}$ pour $p$ suffisamment grand [Yu05]. Nous renvoyons le lecteur à l'introduction de [AL16] pour une présentation du contexte mathématique et des principaux résultats autour des actions affines isométriques propres, et au premier paragraphe pour ce qui concerne les définitions; nous rappelons ici la méthode, bien connue par ailleurs, que nous utilisons pour construire des actions affines.

Une méthode utile pour construire des actions affines. Soit $\left(\mathcal{E}^{\circ},\|\cdot\|\right)$ un espace de Banach et $\pi$ une action continue isométrique d'un groupe topologique $G$ sur $\mathcal{E}^{\circ}$. Supposons donné un espace vectoriel $V$, un plongement d'espace vectoriel de $\mathcal{E}^{\circ}$ dans $V$ et une action linéaire de $G$ sur $V$ qui stabilise $\mathcal{E}^{\circ}$ et dont la restriction à $\mathcal{E}^{\circ}$ est $\pi$. Si $\xi_{\circ}$ est un élément de $V$ tel que, pour tout $g$ de $G$, le vecteur $\xi_{\circ}-g \cdot \xi_{\circ}$ appartient au sous-espace $\mathcal{E}^{\circ}$ et $g \longmapsto \xi_{\circ}-g \cdot \xi_{\circ}$ est continue de $G$ dans $\mathcal{E}^{\circ}$, alors l'action linéaire de $G$ sur $V$ se restreint en une action continue affine isométrique de $G$ sur l'espace de Banach affine $\mathcal{E}=\xi_{\circ}+\mathcal{E}^{\circ}$. Cette dernière est aussi donnée par le cocycle $g \longmapsto \xi_{\circ}-g \cdot \xi_{\circ}$. Par définition, l'action est propre si et seulement si $\lim _{g \rightarrow \infty}\left\|\xi_{0}-g \cdot \xi_{0}\right\|=\infty$.

Comme exemple immédiat d'application de cette méthode, nous en déduisons dans [AL16] que tout groupe discret $G$ de type fini admet une action affine isométrique propre sur l'espace de Banach affine $d(1, \cdot)+\ell^{\infty}(G)$, le cocycle étant dans ce cas donné par la fonction $c(g)=d(1, \cdot)-d(g, \cdot)$, où $d$ désigne la métrique des mots une fois fixé un système fini de générateurs.

Proposition 1.1. Tout groupe discret $G$ de type fini admet une action affine isométrique propre sur un quotient d'un espace de Banach $\ell^{1} d^{\prime}$ une réunion finie de copies de $G$.

Démonstration. Commençons par rappeler que si $\mathcal{G}=\left(\mathcal{G}^{0}, \mathcal{G}^{1}\right)$ est un graphe orienté connexe, où $\mathcal{G}^{0}$ et $\mathcal{G}^{1}$ désignent les ensembles de sommets et d'arêtes, on a un opérateur bord

$$
\partial: \ell^{1}\left(\mathcal{G}^{1}\right) \longrightarrow \mathcal{F}\left(\mathcal{G}^{0}\right),
$$

où $\mathcal{F}\left(\mathcal{G}^{0}\right)$ désigne l'espace vectoriel des fonctions réelles sur les sommets. Précisons ce point. Par définition, $\ell^{1}\left(\mathcal{G}^{1}\right)$ est la complétion pour la norme $\|\cdot\|_{1}$ de l'espace vectoriel $\mathcal{F}_{f}\left(\mathcal{G}^{1}\right)$ des fonctions à support fini sur les arêtes de $\mathcal{G}$. L'image par l'opérateur bord d'une arête $x \rightarrow y$ est par définition la 0-chaîne $\delta_{y}-\delta_{x}$ qui est une fonction (à support fini) de somme nulle sur les sommets de $\mathcal{G}$. Par linéarité, 
$\partial$ s'étend en une application linéaire de $\mathcal{F}_{f}\left(\mathcal{G}^{1}\right)$ dans l'espace vectoriel $\mathcal{F}_{f}^{0}\left(\mathcal{G}^{0}\right)$ des fonctions à support fini et de somme nulle sur les sommets. De plus, cette application est surjective : étant donné deux sommets $x$ et $y$ de $\mathcal{G}$, la fonction $\delta_{y}-\delta_{x}$ est l'image de n'importe quel chemin d'arêtes reliant $x$ à $y$. On peut ainsi définir une norme image sur $\mathcal{F}_{f}^{0}\left(\mathcal{G}^{0}\right)$ qui se calcule facilement sur une fonction de la forme $\delta_{y}-\delta_{x}$ : par définition, c'est l'infimum des normes $\|\cdot\|_{1}$ des fonctions $c$ de $\mathcal{F}_{f}\left(\mathcal{G}^{1}\right)$ telles que $\partial(c)=\delta_{y}-\delta_{x}$. Il n'est pas difficile de voir que pour calculer cet infimum il suffit de considérer les fonctions caractéristiques des chemins d'arêtes reliant $x$ à $y$ (pour une démonstration complète de ce point, on pourra se reporter au lemme 2.8), et qu'on obtient ainsi la distance dans le graphe entre $x$ et $y$. Par complétion des espaces de fonctions à support fini, on en déduit finalement que l'opérateur bord s'étend en une application linéaire bornée surjective de $\ell^{1}\left(\mathcal{G}^{1}\right)$ dans la complétion pour la norme image de $\|\cdot\|_{1}$ de $\mathcal{F}_{f}^{0}\left(\mathcal{G}^{0}\right)$.

Dans le cas où $\mathcal{G}$ est le graphe de Cayley de $G$ associé à un système fini de générateurs, l'action naturelle de $G$ sur $\mathcal{G}^{0}$ induit une action isométrique $\pi$ de $G$ sur l'espace de Banach $\mathcal{E}^{\circ}$ obtenu par complétion pour la norme image de $\|\cdot\|_{1}$ de l'espace vectoriel $\mathcal{F}_{f}^{0}\left(\mathcal{G}^{0}\right)$ des fonctions à support fini et de somme nulle sur les sommets. L'action affine de $G$ sur l'espace affine des fonctions à support fini et de somme 1 sur les sommets induit alors par cette complétion une action affine isométrique de partie linéaire $\left(\pi, \mathcal{E}^{\circ}\right)$.

Nous allons maintenant expliciter cette action affine isométrique de $G$ dans le cadre de la méthode rappelée ci-dessus pour construire des actions affines. En prenant $\xi_{0}=\delta_{1}$ la masse de Dirac en l'élément neutre de $G, \mathcal{E}^{0}=\partial\left(\ell^{1}\left(\mathcal{G}^{1}\right)\right)$ comme espace de Banach et $V=\mathcal{F}\left(\mathcal{G}^{0}\right)$ comme espace vectoriel, on retrouve l'action affine isométrique ci-dessus en tant qu'action affine isométrique de $G$ sur l'espace de Banach affine $\delta_{1}+\partial\left(\ell^{1}\left(\mathcal{G}^{1}\right)\right)$ donnée par le cocycle $g \longmapsto \delta_{1}-\delta_{g}$. La norme de celui-ci n'est autre que la distance de $g$ à l'élément neutre du groupe, donc l'action est propre. Il ne reste plus qu'à noter que le $G$-espace des arêtes $\mathcal{G}^{1}$ s'identifie à $^{\prime}$ une réunion finie de copies de $G$. En effet, pour tout entier naturel $R, l^{\prime}$ application $(x, y) \longmapsto\left(x, x^{-1} y\right)$ de $X^{\leq R}=\{(x, y) \in G \times G ; d(x, y) \leq R\}$ dans $G \times B(1, R)$ est une bijection $G$-équivariante, où l'action de $G \operatorname{sur} G \times B(1, R)$ est par translation à gauche sur le premier facteur ${ }^{1}$. En particulier, $\mathcal{G}^{1}$ s'identifie à $G \times S(1,1)$.

Nous verrons dans la suite de cet article comment étendre la proposition 1.1 à des quotients d'espaces $\ell^{p}$ dès lors que $p$ est suffisamment proche de 1 dans le cas des groupes hyperboliques. Notons dès à présent qu'il est nécessaire de considérer des quotients des espaces $\ell^{p}$, et non pas les espaces $\ell^{p}$ eux-mêmes, pour des $p$ proches de 1 . En effet, d'après Nowak [N09], admettre une action affine isométrique propre sur un espace $\ell^{p}$ pour $1<p<2$ est une caractérisation de la propriété de Haagerup, et l'on sait bien que certains groupes hyperboliques ont la propriété (T) de Kazhdan (par exemple les réseaux de $\operatorname{Sp}(n, 1)$ [G87]).

\section{2. ÉNONCÉ ET DÉMONSTRATION DU THÉORÈME PRINCIPAL}

Nous rappelons la définition d'une classe d'espaces hyperboliques d'un type particulier (contenant les graphes de Cayley des groupes hyperboliques) et nous renvoyons le lecteur au paragraphe 3 de [AL16] pour les définitions et exemples de base à propos des espaces hyperboliques.

Définition 2.1. Un bon espace hyperbolique discret est un espace hyperbolique, uniformément localement fini dont la métrique provient d'une structure de graphe.

1. On désigne par $B(1, R)$ (respectivement $S(1, R))$ la boule (resp. la sphère) de centre 1 et de rayon $R$. 
Notons que puisque la métrique $d: X \longrightarrow \mathbf{N}$ provient d'une structure de graphe, un bon espace hyperbolique discret $(X, d)$ est géodésique dans le sens que, pour tous $x, y$ de $X$ et tout entier $k$ de $\llbracket 0, \cdots, d(a, b) \rrbracket$, il existe un élément $z$ de $X$ tel que $d(x, z)=k$ et $d(z, y)=d(x, y)-k$. Par ailleurs, $d$ étant géodésique, l'uniforme locale finitude équivaut au fait que le nombre de points à distance $1 \mathrm{~d}$ 'un élément donné de $X$ est borné indépendamment de ce point.

Exemple fondamental : si $\Gamma$ est un groupe hyperbolique et $d$ la distance invariante à gauche associée à la longueur des mots pour un système fini de générateurs donné, alors $(\Gamma, d)$ est un bon espace hyperbolique discret; en outre ce dernier est muni d'une action isométrique de $\Gamma$ par translation à gauche ( $d$ provient de la structure de graphe de Cayley sur $\Gamma$ associée au système de générateurs donné).

Notations. Si $\eta$ est un réel positif et $x, y$ sont deux points de l'espace métrique $X$, on désigne par $\eta$-géod $(x, y)$ l'ensemble des points $z$ de $X$ tels que

$$
d(x, z)+d(z, y) \leq d(x, y)+\eta .
$$

On note $\operatorname{géod}(x, y)$ au lieu de 0 -géod $(x, y)$ l'ensemble des points $z$ de $X$ tels que $d(x, z)+d(z, y)=d(x, y)$. Pour tout entier naturel $n$, un chemin de $x$ à $y$ de longueur $n$ est la donnée d'une suite finie de points $\left(x_{i}\right)_{0 \leq i \leq n}$ de $X$ telle que $x_{0}=x, x_{n}=y$ et $d\left(x_{i}, x_{i+1}\right)=1$ pour tout $i$ dans $\llbracket 0, n-1 \rrbracket$; si de plus $x_{0}=x_{n}$, nous appellerons un tel chemin un lacet. Enfin, convenons d'appeler arêtes orientées les éléments de $X^{1}=\{(x, y) \in X \times X ; d(x, y)=1\} ;$ on désigne par $e^{-}$le sommet origine $x$ d'une telle arête $e=(x, y)$ de $X^{1}$, par $e^{+}$son sommet terminal $y$ et par $e^{\text {op }}=(y, x)$ l'arête opposée.

Nous énonçons à présent le théorème principal de cet article.

Théorème 2.2. Soit $(X, d)$ un bon espace hyperbolique discret muni d'une action continue isométrique propre d'un groupe topologique G. Il existe un réel $p_{0}$ strictement supérieur à 1 tel que, pour tout réel $p$ appartenant à $\left[1, p_{0}[, G\right.$ admet une action continue affine isométrique propre sur un quotient de l'espace de Banach $\ell^{p}\left(X^{1}\right)$.

Puisqu'un groupe hyperbolique est en particulier un bon espace hyperbolique discret dont l'espace des arêtes s'identifie à une réunion finie de copies du groupe, le corollaire suivant est une conséquence immédiate du théorème 2.2.

Corollaire 2.3. Pour tout groupe hyperbolique $\Gamma$, il existe un entier naturel $k$ non nul tel que, en notant $\Gamma_{k}$ la réunion disjointe de $k$ copies de $\Gamma$, pour tout $p$ suffisamment proche de $1, \Gamma$ admet une action affine isométrique propre sur un quotient de l'espace de Banach $\ell^{p}\left(\Gamma_{k}\right)$.

La suite de cet article est consacrée à la démonstration du théorème 2.2 dont la stratégie de preuve reprend celle de la proposition 1.1 : le cœur du problème est de démontrer qu'un certain cocycle, analogue au cocycle introduit dans la proposition 1.1, est propre, ce qui n'est pas vrai en général pour $p>1$ sans hypothèse sur l'espace métrique $(X, d)$. Nous allons utiliser de manière cruciale l'hypothèse d'hyperbolicité qui implique qu'un chemin entre deux points $x$ et $y$ de $(X, d)$ reste proche de toute géodésique entre ces deux points. Une formulation quantitative de cette heuristique est donnée par la proposition 2.6 dont on voit bien qu'elle tombe en défaut pour un espace métrique comme $\left(\mathbf{Z}^{2}, d_{\text {eucl. }}\right)$.

Démonstration. Le groupe $G$ opère naturellement dans l'espace vectoriel $\mathcal{F}_{f}\left(X^{1}\right)$ des fonctions réelles à support fini sur $X^{1}=\{(x, y) \in X \times X ; d(x, y)=1\}$, ainsi que dans l'espace vectoriel $\mathcal{F}_{f}^{0}(X)$ des fonctions réelles à support fini et de somme nulle sur $X$. Par ailleurs l'application linéaire bord $\partial: \mathcal{F}_{f}\left(X^{1}\right) \longrightarrow \mathcal{F}_{f}^{0}(X)$, définie par $\partial \delta_{(x, y)}=\delta_{y}-\delta_{x}$ est surjective et $G$-équivariante. Pour $p$ dans $] 1 ; \infty[$, on munit l'espace 
vectoriel $\mathcal{F}_{f}\left(X^{1}\right)$ des fonctions réelles à support fini sur $X^{1}$ de la norme $\ell^{p}$ notée $\|\cdot\|_{p}$. L'action de $G$ étant isométrique, après complétion des espaces de fonctions ci-dessus, on en déduit une action continue affine isométrique de G sur l'espace de Banach affine obtenu par complétion pour la norme image de $\|\cdot\|_{p}$ par $\partial$ de l'espace affine $\mathcal{F}_{f}^{1}(X)$ des fonctions à support fini et de somme 1 sur $X$. Il reste alors à voir que le cocycle défini par cette action est propre, pour tout $p$ suffisamment proche de 1 , ce que nous déduirons de la proposition 2.9. Pour prouver cette dernière, nous démontrerons d'abord la proposition 2.6 et son corollaire 2.7.

Soit $(X, d)$ un bon espace hyperbolique discret et $\delta$ une constante d'hyperbolicité de $X$ que l'on supposera strictement positive. Commençons par démontrer le lemme d'emboîtement des géodésiques, lemme bien connu et qui nous sera utile par la suite.

Lemme 2.4. Soit $a, b, c, d$ quatre points de X. Si b appartient à $\eta_{1}$-géod $(a, c)$, si c appartient à $\eta_{2}$-géod $(b, d)$ et si $d(b, c)>\left(\eta_{1}+\eta_{2}+\delta\right) / 2$, alors b appartient à $\left(\eta_{1}+\delta\right)$-géod $(a, d)$ et c à $\left(\eta_{2}+\delta\right)-\operatorname{géod}(a, d)$.

Démonstration. Par définition de la $\delta$-hyperbolicité de $X$ ([AL16], déf. 3.2), on a

$$
d(a, c)+d(b, d) \leq \max (d(a, d)+d(b, c), d(a, b)+d(c, d))+\delta .
$$

Puisque $b$ appartient à $\eta_{1}$-géod $(a, c)$ (i.e. $\left.d(a, b)+d(b, c) \leq d(a, c)+\eta_{1}\right)$, on en déduit donc

$$
d(a, b)+d(b, c)+d(b, d) \leq \max (d(a, d)+d(b, c), d(a, b)+d(c, d))+\eta_{1}+\delta,
$$

ou encore

$$
d(a, b)+d(b, d) \leq \max (d(a, d), d(a, b)+d(c, d)-d(b, c))+\eta_{1}+\delta .
$$

En utilisant à présent que $c$ appartient à $\eta_{2}$-géod $(b, d)\left(\right.$ i.e. $\left.d(b, c)+d(c, d) \leq d(b, d)+\eta_{2}\right)$, on en déduit alors

$$
d(a, b)+d(b, d) \leq \max \left(d(a, d), d(a, b)+d(b, d)-2 d(b, c)+\eta_{2}\right)+\eta_{1}+\delta .
$$

Comme $d(b, c)>\left(\eta_{1}+\eta_{2}+\delta\right) / 2$, on en conclut finalement que

$$
d(a, b)+d(b, d) \leq d(a, d)+\eta_{1}+\delta
$$

On démontre de même que $c$ appartient à $\left(\eta_{2}+\delta\right)$-géod $(a, d)$ en appliquant la première partie du lemme que nous venons de démontrer au quadruplet $(d, c, b, a)$.

Étant donné un point $t$ dans $X$, on note $(\cdot, \cdot)_{t}$ le produit de Gromov par rapport à $t$, fonction définie sur $X \times X$ par

$$
(x, y)_{t}=\frac{1}{2}(d(x, t)+d(y, t)-d(x, y))
$$

Rappelons que, pour tous points $x$ et $y$ de $X$ et pour tout segment géodésique $\gamma_{x y}$ entre ces deux points, la différence $d\left(t, \gamma_{x y}\right)-(x, y)_{t}$ entre la distance de $t$ à $\gamma_{x y}$ et le produit de Gromov de $x, y$ par rapport à $t$ est, en valeur absolue, majorée par une constante ne dépendant que de $\delta$. Par ailleurs, par définition de la $\delta$ hyperbolicité de $X$ ([GdlH90], chap. 2, déf. 3), pour tous points $x, y, z$ et $t$ de $X$, en notant $K=\mathrm{e}^{\delta} \geq 1$, on a

$$
\mathrm{e}^{-(x, y)_{t}} \leq K \cdot \max \left\{\mathrm{e}^{-(x, z)_{t}}, \mathrm{e}^{-(z, y)_{t}}\right\}
$$

Un argument analogue à la proposition $10 \mathrm{du}$ chapitre $7 \mathrm{de}$ [GdlH90] permet de démontrer qu'il existe un réel $\epsilon>0$ et un réel $C>1$ tels que, en tout point $t$ de $X$, 
il existe une fonction $d_{t}$ définie sur $X \times X$ satisfaisant l'inégalité triangulaire et vérifiant, pour tous points $x$ et $y$ de $X$,

$$
\frac{1}{C} \cdot \mathrm{e}^{-\epsilon \cdot(x, y)_{t}} \leq d_{t}(x, y) \leq C \cdot \mathrm{e}^{-\epsilon \cdot(x, y)_{t}} .
$$

Convenons d'appeler une telle fonction $d_{t}$ une métrique visuelle centrée en $t$, de paramètre visuel $\mathrm{e}^{\epsilon}$ et de constante $C$. Notons que, si $t$ appartient à $\eta$-géod $(x, y)$, alors les deux points $x$ et $y$ sont à une distance visuelle $d_{t}(x, y)$ l'un de l'autre au moins égale à $\mathrm{e}^{-\epsilon \cdot \eta / 2} / C$. Remarquons enfin que, pour tout $x$ de $X, d_{t}(x, x)>0$, ce qui fait que $d_{t}$ n'est pas une métrique au sens strict du terme.

Lemme 2.5. Soit $(X, d)$ un bon espace hyperbolique discret. Il existe deux réels $\epsilon$ et $\alpha_{\eta}$ strictement positifs tels que pour tout chemin $\left(x_{i}\right)_{0 \leq i \leq n}$ de longueur $n \geq 1$, si t appartient à $\eta$-géod $\left(x_{0}, x_{n}\right)$, alors

$$
\sum_{i=0}^{n-1} e^{-\epsilon \cdot d\left(x_{i}, t\right)} \geq \alpha_{\eta}
$$

Démonstration. Soit $\left(x_{i}\right)_{0 \leq i \leq n}$ un chemin de longueur $n, t$ un point de $\eta$-géod $\left(x_{0}, x_{n}\right)$ et $d_{t}$ une métrique visuelle sur $X$, centrée en $t$, de paramètre visuel $\mathrm{e}^{\epsilon}$ et de constante $C$. On a alors les inégalités suivantes :

$$
C \cdot \sum_{i=0}^{n-1} \mathrm{e}^{-\epsilon \cdot\left(x_{i}, x_{i+1}\right)_{t}} \geq \sum_{i=0}^{n-1} d_{t}\left(x_{i}, x_{i+1}\right) \geq d_{t}\left(x_{0}, x_{n}\right) \geq \mathrm{e}^{-\epsilon \cdot \eta / 2} / C .
$$

En effet, la première inégalité provient de la majoration satisfaite par la métrique visuelle $d_{t}$ et la deuxième inégalité est l'inégalité triangulaire pour cette même métrique visuelle. Quant à la dernière inégalité, elle résulte du fait que $t$ appartient à $\eta$-géod $\left(x_{0}, x_{n}\right)$.

Par ailleurs, on déduit facilement du produit de Gromov et du fait que $x_{i}$ et $x_{i+1}$ sont deux points voisins dans $X$ l'encadrement suivant :

$$
d\left(x_{i}, t\right)-1 \leq\left(x_{i}, x_{i+1}\right)_{t} \leq d\left(x_{i}, t\right) .
$$

En utilisant les inégalités 1 et 2 ci-dessus, on en déduit finalement l'inégalité annoncée avec $\alpha_{\eta}=\mathrm{e}^{-\epsilon(\eta / 2+1)} / C^{2}$.

Proposition 2.6. Soit $(X, d)$ un bon espace hyperbolique discret. Il existe deux réels $\epsilon$ et $\beta$ strictement positifs tels que pour tous points $x$ et $y$ dans $X$ et tout chemin $\left(x_{i}\right)_{0 \leq i \leq n}$ tel que $x_{0}=x$ et $x_{n}=y$, on $a$

$$
\sum_{i=0}^{n-1} e^{-\epsilon \cdot d\left(x_{i}, g e ́ o d(x, y)\right)} \geq \beta \cdot d(x, y)
$$

Si $X$ est un arbre, la proposition précédente est facilement vérifiée quel que soit le choix de $\epsilon$ strictement positif avec $\beta=1$, puisque tout chemin de $x$ à $y$ contient le segment $\operatorname{géod}(x, y)$. Par contre, on vérifie sans peine que cette proposition est fausse dans le cas du plan euclidien $\left(\mathbf{Z}^{2}, d_{\text {eucl. }}\right)$. En effet, en notant $d$ la distance entre deux points $x$ et $y$ de $\mathbf{Z}^{2}$ situés sur une même horizontale et en considérant un chemin « rectangulaire» de longueur $n=2 m+d$ entre $x$ et $y$ comme indiqué sur la figure ci-dessous, on calcule facilement la distance d'un point de ce chemin au segment horizontal inférieur qui est le segment géodésique reliant $x$ et $y$. 


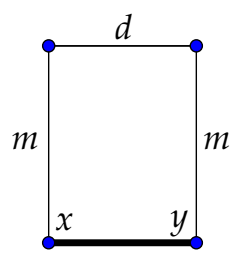

Les $d$ points du segment horizontal supérieur sont tous à distance $m$ du segment géodésique entre $x$ et $y$, et les points des segments verticaux sont à une distance de $x$ ou $y$ variant de 0 à $m-1$. On en déduit donc

$$
\sum_{i=0}^{n-1} \mathrm{e}^{-\epsilon \cdot d_{\text {eucl }}\left(x_{i}, g \text { ǵod }(x, y)\right)}=\sum_{k=0}^{m-1} \mathrm{e}^{-\epsilon \cdot k}+d \cdot \mathrm{e}^{-\epsilon \cdot m}+\sum_{k=1}^{m} \mathrm{e}^{-\epsilon \cdot k} \leq \frac{2}{1-\mathrm{e}^{-\epsilon}}+d \cdot \mathrm{e}^{-\epsilon \cdot m},
$$

ce qui interdit à la somme considérée dans la proposition 2.6 d'être minorée par $\beta \cdot d$ pour tous $d$ et $m$, dès lors que $\beta$ et $\epsilon$ sont strictement positifs.

Démonstration. Soit $x$ et $y$ deux points de $X$ et $\left(x_{i}\right)_{0 \leq i \leq n}$ un chemin de $x$ à $y$.

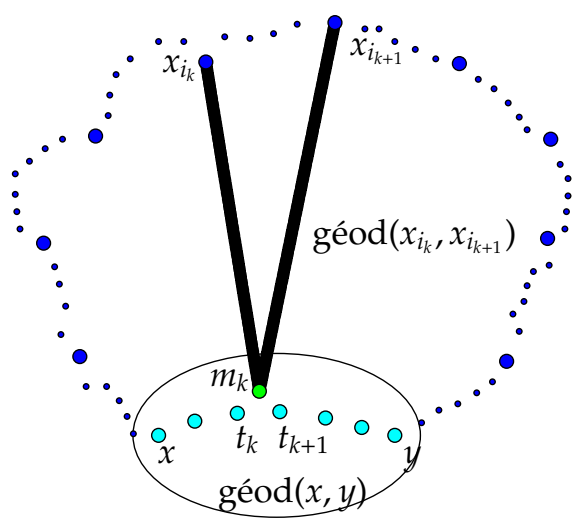

Pour tout $i$ de $[[0, n-1]]$, la distance entre tout point de géod $(x, y)$ parmi les plus proches de $x_{i}$ et tout point de géod $(x, y)$ parmi les plus proches de $x_{i+1}$ est inférieure à $\kappa \cdot \delta$, où $\kappa$ est une constante ne dépendant que de $(X, \delta)$. Par conséquent il est possible de fixer deux constantes $\delta_{1}$ et $\delta_{2}$ (ne dépendant que de $\delta$ ) vérifiant $\delta_{2} \geq \delta_{1}>3 \delta+1$ telles que si la distance entre $x$ et $y$ est supérieure à $\delta_{1}$, on peut extraire une sous-suite $\left(x_{i_{k}}\right)_{0 \leq k \leq l}$ du chemin $\left(x_{i}\right)_{0 \leq i \leq n}$ et une suite de points $\left(t_{k}\right)_{0 \leq k \leq l}$ de géod $(x, y)$ telles que $x_{i_{0}}=t_{0}=x, x_{i_{l}}=t_{l}=y$, et pour tout $k$ dans $[[0, l-1]]$ :

- $t_{k}$ est un point de géod $(x, y)$ parmi les plus proches de $x_{i_{k}}$ (autrement dit, $d\left(x_{i_{k}}, t\right) \geq d\left(x_{i_{k}}, t_{k}\right)$ pour tout point $t$ de géod $\left.(x, y)\right)$;

- la distance entre $t_{k}$ et $t_{k+1}$ est contrôlée par $\delta_{1}$ et $\delta_{2}: \delta_{1} \leq d\left(t_{k}, t_{k+1}\right) \leq \delta_{2}$.

Si la distance entre $x$ et $y$ est strictement inférieure à $\delta_{1}$, la proposition résulte du lemme 2.5. Sinon on applique l'argument suivant.

Soit $m_{k}$ un milieu ${ }^{2}$ de la paire de points $\left\{t_{k}, t_{k+1}\right\}$ pour laquelle on note $D=$ $d\left(t_{k}, t_{k+1}\right)$. Une première application du lemme $2.4 \mathrm{~d}^{\prime}$ emboîtement des géodésiques au quadruplet de points $\left(x_{i_{k}}, t_{k}, m_{k}, t_{k+1}\right)$ (avec $a=x_{i_{k}}, b=t_{k}, c=m_{k}, d=t_{k+1}, \eta_{1}=$ $\left.d\left(t_{k}, m_{k}\right), \eta_{2}=0\right)$ permet d'affirmer que $t_{k}$ appartient à $\Delta$-géod $\left(x_{i_{k}}, t_{k+1}\right)$ avec $\Delta=$

2. Convenons de désigner par milieu d'une paire de points $\{x, y\}$ de $X$ un point $z$ de géod $(x, y)$ tel que $|d(x, z)-d(y, z)| \leq 1$. 
$(D+1) / 2+\delta$, puisque

$$
d\left(t_{k}, m_{k}\right)>\frac{\eta_{1}+\eta_{2}+\delta}{2}=d\left(t_{k}, m_{k}\right) / 2+\delta / 2
$$

ce qui suit de $\delta_{1}>\delta$. De manière symétrique, une deuxième application du même lemme au quadruplet $\left(t_{k}, m_{k}, t_{k+1}, x_{i_{k+1}}\right)$ assure cette fois-ci que $t_{k+1}$ appartient à $\Delta$-géod $\left(t_{k}, x_{i_{k+1}}\right)$.

Une troisième et dernière application du lemme 2.4 au quadruplet $\left(x_{i_{k}}, t_{k}, t_{k+1}, x_{i_{k+1}}\right)$ permet de déduire que $t_{k}$ et $t_{k+1}$ appartiennent à $\Delta^{\prime}-\operatorname{géod}\left(x_{i_{k}}, x_{i_{k+1}}\right)$ avec $\Delta^{\prime}=\Delta+\delta$, puisque

$$
d\left(t_{k}, t_{k+1}\right)=D>\frac{2 \Delta+\delta}{2}=D / 2+(3 \delta+1) / 2
$$

ce qui suit de $\delta_{1}>3 \delta+1$.

On en déduit finalement que $m_{k}$ appartient à $\Delta^{\prime \prime}-\operatorname{géod}\left(x_{i_{k}}, x_{i_{k+1}}\right)$ avec $\Delta^{\prime \prime}=\Delta^{\prime}+$ $D+1=3(D+1) / 2+2 \delta$. En effet,

$$
d\left(x_{i_{k}}, m_{k}\right)+d\left(m_{k}, x_{i_{k+1}}\right) \leq d\left(x_{i_{k}}, t_{k}\right)+d\left(t_{k}, x_{i_{k+1}}\right)+2 d\left(t_{k}, m_{k}\right) \leq d\left(x_{i_{k}}, x_{i_{k+1}}\right)+\Delta^{\prime \prime} .
$$

Or d'après le lemme 2.5 (avec $x=x_{i_{k}}, y=x_{i_{k+1}}, t=m_{k}, \eta=\Delta^{\prime \prime}$ ), il existe deux réels $\epsilon$ et $\alpha$ strictement positifs tels que, pour tout $k$,

$$
\sum_{i=i_{k}}^{i_{k+1}-1} \mathrm{e}^{-\epsilon \cdot d\left(x_{i}, \operatorname{géod}(x, y)\right)} \geq \sum_{i=i_{k}}^{i_{k+1}-1} \mathrm{e}^{-\epsilon \cdot d\left(x_{i}, m_{k}\right)} \geq \alpha .
$$

On en déduit finalement l'inégalité annoncée en posant $\beta=\alpha / \delta_{2}$ puisqu'alors

$$
\sum_{i=0}^{n-1} \mathrm{e}^{-\epsilon \cdot d\left(x_{i}, \operatorname{gé} \mathrm{d}(x, y)\right)}=\sum_{k=0}^{l-1} \sum_{i=i_{k}}^{i_{k+1}-1} \mathrm{e}^{-\epsilon \cdot d\left(x_{i}, \operatorname{géod}(x, y)\right)} \geq \alpha \cdot d(x, y) / \delta_{2} .
$$

On appelle chaîne toute combinaison linéaire (finie) $c$ d'arêtes orientées, avec la relation que l'arête opposée $e^{\mathrm{op}} \mathrm{d}^{\prime}$ une arête orientée $e$ est égale à $-e$. Autrement dit, une chaîne est un élément du quotient de l'espace vectoriel libre engendré par les arêtes orientées modulo la relation $e^{\mathrm{op}}+e=0$ pour toute arête orientée $e$; on note $\bar{e}$ l'image dans le quotient d'une telle arête orientée $e$. On note $c(e)$ la différence des coefficients de $\bar{e}$ et $\overline{e^{\mathrm{op}}}$ dans $c$, de sorte que $c \mapsto c(e)$ est la forme linéaire sur ce quotient qui envoie $\bar{e}$ sur $1, \overline{e^{\mathrm{op}}}$ sur -1 , et les autres arêtes orientées sur 0 . On remarque que $c(e) \cdot \bar{e}$ ne dépend pas de l'orientation de $e(\operatorname{car} c(e)$ et $\bar{e}$ changent tous les deux de signe quand on remplace $e$ par $\left.e^{\mathrm{op}}\right)$. On a alors $c=\sum_{e / \sim} c(e) \cdot \bar{e}$, où la somme porte sur les arêtes modulo l'orientation. Enfin, nous dirons qu'une arête $e$ est dans le support de $c$ si $c(e)$ est non nul.

Corollaire 2.7. Pour toute chaîne $c=\sum_{e / \sim} c(e) \cdot \bar{e}$ de bord $\delta_{y}-\delta_{x}$, on a

$$
\sum_{e / \sim}|c(e)| e^{-\epsilon \cdot d(e, g e ́ o d(x, y))} \geq \beta \cdot d(x, y),
$$

où $d(e$, géod $(x, y))=\min \left(d\left(e^{-}\right.\right.$, géod $\left.(x, y)\right), d\left(e^{+}\right.$, géod $\left.\left.(x, y)\right)\right)$.

Pour démontrer le corollaire 2.7, nous aurons besoin du lemme suivant. Nous dirons qu'une chaîne est un cycle si son bord est nul.

Lemme 2.8. Pour toute chaîne $c=\sum_{e / \sim} c(e) \cdot \bar{e}$ de bord $\delta_{y}-\delta_{x}$, il existe des coefficients réels $\alpha_{k}$ non nuls, des chemins $c_{k}$ de bord $\delta_{y}-\delta_{x}$ et un cycle $\ell=\sum_{j} \beta_{j} \cdot l_{j}$, où les $\beta_{j}$ sont des coefficients strictement positifs et les $l_{j}$ des lacets, tels que $c=\sum_{k} \alpha_{k} \cdot c_{k}+\ell, \sum_{k} \alpha_{k}=1$ et

$$
\|c\|_{1}=\sum_{e / \sim}|c(e)|=\sum_{k}\left|\alpha_{k}\right| \cdot\left\|c_{k}\right\|_{1}+\|\ell\|_{1} .
$$


Démonstration. Soit $c=\sum_{e / \sim} c(e) \cdot \bar{e}$ une chaîne de bord $\delta_{y}-\delta_{x}$. On note $\mathcal{S}_{c}$ le graphe orienté formé des arêtes $e$ telles que $c(e)$ est strictement positif : c'est un raffinement du support de $c$ tenant compte de l'orientation.

Si c est nulle, il n'y a rien à démontrer. On suppose donc $c$ non nulle.

Puisque le bord de $c$ est $\delta_{y}-\delta_{x}$, pour tout sommet $z$ distinct de $x$ ou $y$, la somme des coefficients $c(e)$ des arêtes $e$ de sommet terminal $z$ dans le graphe $\mathcal{S}_{c}$ est égale à la somme des coefficients $c(e)$ des arêtes $e$ de sommet origine $z^{3}$. En particulier, si $e$ est une arête de $\mathcal{S}_{c}$ de sommet terminal $z$ distinct de $x$ ou $y$, alors il existe une arête $e^{\prime}$ dans $\mathcal{S}_{c}$ de sommet origine $z$. Ainsi, partant du sommet $x$, on construit, arête après arête, un chemin $c_{1}$ d'origine $x$ et d'extrémité $y$ dans $\mathcal{S}_{c}$, ou bien un lacet $l_{1}$ si le chemin se recoupe.

Selon le cas, on note $\alpha_{1}>0$ (respectivement $\beta_{1}$ ) le minimum des $c_{1}(e)$ pour les arêtes $e$ dans le support de $c_{1}$ (resp. $l_{1}$ ). On considère alors la chaîne $c^{\prime}=c-\alpha_{1} \cdot c_{1}$ (resp. $\left.c-\beta_{1} \cdot l_{1}\right)$ de bord $\left(1-\alpha_{1}\right) \cdot\left(\delta_{y}-\delta_{x}\right)$ (resp. de même bord que $c$ ) et de norme $\ell^{1}$ égale à $\|c\|_{1}-\alpha_{1} \cdot\left\|c_{1}\right\|_{1}$ (resp. $\|c\|_{1}-\beta_{1} \cdot\left\|l_{1}\right\|_{1}$ ). Par construction, le cardinal du support de $c^{\prime}$ est strictement inférieur à celui de $c$. Si le bord de $c^{\prime}$ est nul, $c^{\prime}$ est terminé. Sinon on réitère le raisonnement précédent avec la chaîne $\frac{1}{1-\alpha_{1}} c^{\prime}$ (resp. $c^{\prime}$ ) au lieu de $c$. On en déduit le résultat après un nombre fini d'itérations.

Nous démontrons à présent le corollaire 2.7.

Démonstration. Soit $c=\sum_{e / \sim} c(e) \cdot \bar{e}$ une chaîne de bord $\delta_{y}-\delta_{x}$. D'après le lemme 2.8, il existe des chemins $c_{k}$ de bord $\delta_{y}-\delta_{x}$, des réels non nuls $\alpha_{k}$ et un cycle $\ell$ tels que

$$
c=\sum_{k} \alpha_{k} \cdot c_{k}+\ell
$$

avec $\sum_{k} \alpha_{k}=1$ et $\|c\|_{1}=\sum_{k}\left|\alpha_{k}\right| \cdot\left\|c_{k}\right\|_{1}+\|\ell\|_{1}$. Pour chaque chemin $c_{k}$ de bord $\delta_{y}-\delta_{x}$, en indexant par $e_{k, j}$ les arêtes d'un tel chemin, la proposition 2.6 assure que

$$
\sum_{j} \mathrm{e}^{-\epsilon \cdot d\left(e_{k, j}^{-}, \operatorname{réod}(x, y)\right)} \geq \beta \cdot d(x, y) .
$$

On en déduit donc

$$
\sum_{e / \sim}|c(e)| \mathrm{e}^{-\epsilon \cdot d(e, g e ́ o d(x, y))} \geq \sum_{k}\left|\alpha_{k}\right| \sum_{j} \mathrm{e}^{-\epsilon \cdot d\left(e_{k, j}^{-}, \operatorname{có}^{\prime} \mathrm{d}(x, y)\right)} \geq \beta \cdot d(x, y) .
$$

Proposition 2.9. Soit $(X, d)$ un bon espace hyperbolique discret. Pour tout $p$ suffisamment proche de 1 , il existe un réel $\alpha^{\prime}$ strictement positif tel que pour toute chaîne $c=\sum_{e / \sim} c(e) \cdot \bar{e}$ de bord $\delta_{y}-\delta_{x}$, on a

$$
\|c\|_{p}=\left(\sum_{e / \sim}|c(e)|^{p}\right)^{1 / p} \geq \alpha^{\prime} \cdot d(x, y)^{1 / p} .
$$

Démonstration. Soit $1<p<\infty$ et $q$ tel que $1 / p+1 / q=1$. L'inégalité de Hölder assure que pour tous $f$ de $\ell^{p}$ et $g$ de $\ell^{q}$, la norme $\|\cdot\|_{1}$ de $f g$ est majorée par le produit $\|f\|_{p}\left\|_{g}\right\|_{q}$. On en déduit donc

$$
\left(\sum_{e / \sim}|c(e)|^{p}\right)^{1 / p}\left(\sum_{e / \sim} \mathrm{e}^{-\epsilon q \cdot d(e, \operatorname{géod}(x, y))}\right)^{1 / q} \geq \sum_{e / \sim}|c(e)| \mathrm{e}^{-\epsilon \cdot d(e, \operatorname{gé} \operatorname{dod}(x, y))} \geq \beta \cdot d(x, y),
$$

3. Si on pense à $\mathcal{S}_{c}$ comme un circuit électrique, on traduit ainsi mathématiquement la loi des nœuds de Kirchhoff qui assure qu'en tout nœud du réseau, la somme des intensités des courants entrants est égale à la somme des intensités des courants sortants. 
la première inégalité résultant de l'inégalité de Hölder et la deuxième du corollaire 2.7. Or, l'espace $(X, d)$ étant uniformément localement fini, le nombre de sommets à distance $k$ de géod $(x, y)$ est au plus à croissance exponentielle en $k$. Plus précisément, il existe un réel $\beta^{\prime}$ strictement positif et une constante multiplicative $D$ tels que, pour $x$ distinct de $y$,

$$
\operatorname{card}(\{z \in X ; d(z, \operatorname{géod}(x, y)) \leq k\}) \leq D \cdot \mathrm{e}^{\beta^{\prime} k} d(x, y),
$$

ce qui permet de donner la minoration suivante

$$
\left(\sum_{e / \sim} \mathrm{e}^{-\epsilon q \cdot d(e, \operatorname{gé} o d(x, y))}\right)^{1 / q} \leq D^{1 / q}\left(\sum_{k=0}^{\infty} \mathrm{e}^{\left(\beta^{\prime}-\epsilon q\right) k}\right)^{1 / q} d(x, y)^{1 / q} .
$$

La série $\sum_{k} \mathrm{e}^{\left(\beta^{\prime}-\epsilon q\right) k}$ ci-dessus est convergente si et seulement si $q>\beta^{\prime} / \epsilon$. Dans ce cas, en notant $D^{\prime}$ la somme de cette série, on en déduit l'énoncé du lemme pour tout $1<p<\beta^{\prime} /\left(\beta^{\prime}-\epsilon\right)$ en posant $\alpha^{\prime}=\beta /\left(D D^{\prime}\right)^{1 / q}$.

Ceci termine la démonstration du théorème 2.2 puisque, étant donné un point $o$ de $X, l^{\prime}$ action de $G$ sur $X$ étant propre, la proposition 2.9 assure alors que le cocycle $g \longmapsto \delta_{o}-\delta_{g \cdot o}$ à valeurs dans $\partial\left(\ell^{p}\left(X^{1}\right)\right)$ est propre pour tout $p$ suffisamment proche de 1 (la continuité du cocycle résultant du fait qu'il est localement constant, comme conséquence de la continuité de $g \longmapsto g \cdot o$ de $G$ dans $X$ discret).

Remerciements : Nous remercions Mikael de la Salle pour de stimulantes discussions et Peter Haissinsky pour des références concernant la métrique visuelle.

\section{RÉFÉRENCES}

[AL16] A. Alvarez et V. Lafforgue. Actions affines isométriques propres des groupes hyperboliques sur des espaces $\ell^{p}$.

Expositiones mathematicae. Vol. 34, p. 236-257 (2016).

[GdlH90] E. Ghys et P. de la Harpe, éditeurs. Sur les groupes hyperboliques d'après Mikhael Gromov. Progress in Mathematics. Vol. 83. Birkhäuser Boston Inc.(1990).

[G87] M. Gromov. Hyperbolic groups.

Math. Sci. Res. Inst. Publ. Vol. 8 of Essays in group theory. Springer, New York (1987).

[N09] P. W. Nowak. Group actions on Banach spaces and a geometric characterization of a-T-menability. Topology and its Applications (2009).

[Yu05] G. Yu. Hyperbolic groups admit proper affine isometric actions on $\ell^{p}$ spaces. Geom. and Funct. Anal. Vol. 15, p. 1144-1151 (2005).

Aurélien Alvarez fait partie du projet ANR-14-CE25-0004 GAMME.

MAPMO, UMR 7349, UNIVERSITÉ D'ORLÉANS

Rue de Chartres, BP 6759 - 45067 Orléans cedex 2, France

VinCENT LAFFORGUE FAIT PARTIE DU PROJET ANR-14-CE25-0012 SINGSTAR.

CNRS et Institut Fourier, UMR 5582, Université Grenoble Alpes

100 rue des Maths, 38610 Gières, France 